\title{
Mathematical Basis for the Plasma Kinetic Equations (BBGKY)
}

\author{
John J. Sopka* \\ Institute for Basic Standards, National Bureau of Standards, Boulder, Colo. 80302
}

(March 29, 1968)

\begin{abstract}
The general family of kinetic equations, which in plasma kinetics are called the BBGKY equations, are obtained rigorously from basic probabilistic considerations in order to exhibit explicitly the conditions or assumptions under which they obtain.
\end{abstract}

Key Words: BBGKY hierarchy equations, probabilistic basis for kinetic equations.

\section{Introduction}

The plasma kinetic equations, referred to as the BBGKY hierarchy, are fundamental in the analysis of plasma kinetics. In the following, these equations are derived rigorously in order to determine explicitly the conditions or assumptions under which they are valid. The heuristic derivations do not clearly indicate the assumptions which are important to both theoretical and experimental plasma research. Since the functions in the BBGKY equations are probability density functions the following treatment is probabilistic.

\section{Preliminaries}

Consider first Euclidean 6-space $E^{6}$ in which a point is represented by the 6-tuple of real numbers (or coordinates) $\left\{q_{1}, q_{2}, q_{3}, p_{1}, p_{2}, p_{3}\right\}$ and $n$ points are represented by $n$ 6-tuples $\left\{q_{1}^{s}\right.$, $\left.q_{2}^{s}, q_{3}^{s}, p_{1}^{s}, p_{2}^{s}, p_{3}^{s}\right\}$ for $s=1 \ldots n$ or more concisely for each $s=1 \ldots n$ by $\left\{q_{i}^{s} p_{i}^{s}\right\}$ where $i=1,2,3$. Equivalently these $n$ points may be represented in Euclidean $6 n$-space $E^{6 n}$ by one point, or $6 n$-tuple, $\left\{q_{i}^{s}, p_{i}^{s}\right\}$ where $s=1 \ldots n, i=1,2,3$.

Consider also a set of $6 n$ elements $Q_{1} \ldots Q_{3 n} P_{1} \ldots P_{3 n}$ called observables; the entire set $Q_{1} \ldots Q_{3 n} P_{1} \ldots P_{3 n}$ being called the System $S$ of observables. We then make the definition: Definition: $A$ (static) state $\mathrm{P}$ of the $S y$ stem $S$ is an assignment $\mathrm{P}: \mathrm{Q}_{1} \ldots \mathrm{Q}_{3 \mathrm{n}} \mathrm{P}_{1} \ldots \mathrm{P}_{3 \mathrm{n}} \rightarrow\left\{\mathrm{q}_{1}^{1}\right.$ . . . $\left.\mathrm{q}_{3}^{\mathrm{n}}, \mathrm{p}_{1}^{1} \ldots \mathrm{p}_{3}^{\mathrm{n}}\right\}$ i.e., an assignment of $6 \mathrm{n}$ numerical values to the observables or an assignment of a point in $\mathrm{E}^{6 \mathrm{n}}$. The correspondence between the $\mathrm{P}^{\prime}$ 's and the points $\left\{\mathrm{q}_{\mathrm{i}}^{\mathrm{s}}, \mathrm{p}_{\mathrm{i}}^{\mathrm{s}}\right\}$ is assumed to be 1:1 so that we may write $\mathrm{P}=\left\{\mathrm{q}_{\mathrm{i}}^{\mathrm{s}}, \mathrm{p}_{\mathrm{i}}^{\mathrm{s}}\right\}$.

The statistics of the System (in this static case) are given by a probability distribution $F$ on the Borel Field of $E^{6 n}$ such that the following assumption holds:

AsSUMPTION 1: F is absolutely continuous with respect to Lebesgue measure and thus there exists a measurable function $\rho=\rho\left(\mathrm{q}_{\mathrm{i}}^{\mathrm{s}}, \mathrm{p}_{\mathrm{i}}^{\mathrm{s}}\right)$; i.e., a probability density function, such that for any set $\mathrm{B}$ in the Borel Field one has:

$$
\mathrm{F}(\mathrm{B})=\int_{\mathrm{B}} \rho\left(\mathrm{q}_{\mathrm{i}}^{\mathrm{s}}, \mathrm{p}_{\mathrm{i}}^{\mathrm{s}}\right)(\mathrm{dq} \mathrm{dp})_{\mathrm{n}} \quad \text { where }(\mathrm{dq} \mathrm{dp})_{\mathrm{n}}=\mathrm{dq}_{1}^{1} \ldots \mathrm{dq}_{3}^{\mathrm{n}} \mathrm{dp}_{1}^{1} \ldots \mathrm{dp}_{3}^{\mathrm{n}} .
$$

${ }^{*}$ Consultant in Mathematics, Radio Standards Laboratory, NBS Laboratories, Boulder, Colo. 80302. 
In order to introduce dynamics into this schema, we specify the state concept in the dynamic case as follows:

Definition: A state function $\mathrm{P}$ of the System $\mathrm{S}$ is a mapping from the direct product $\mathrm{S} \times \mathrm{R} \#$, where $\mathrm{R} \#$ is the real numbers, into $\mathrm{E}^{6 \mathrm{n}}$ such that for each $\mathrm{t} \epsilon \mathrm{R} \#, \mathrm{P}$ provides an assignment $\mathrm{P}(\mathrm{t})$ of a point in $\mathrm{E}^{6 \mathrm{n}}$ to the observables in $\mathrm{S}$, that is, for each $\mathrm{t}, \mathrm{P}$ specifies a (static) state $\mathrm{P}(\mathrm{t}): \mathrm{Q}_{1} \ldots \mathrm{Q}_{3 \mathrm{n}}$ $\mathrm{P}_{1} \ldots \mathrm{P}_{3 \mathrm{n}} \rightarrow\left\{\mathrm{q}_{\mathrm{i}}^{\mathrm{s}}(\mathrm{t}), \mathrm{p}_{\mathrm{i}}^{\mathrm{s}}(\mathrm{t})\right\}$ which by the preceding convention we may write $\mathrm{P}(\mathrm{t})=\left\{\mathrm{q}_{\mathrm{i}}^{\mathrm{s}}(\mathrm{t}), \mathrm{p}_{\mathrm{i}}^{\mathrm{s}}(\mathrm{t})\right\}$.

We shall also make the following assumptions:

Assumption 2: For given $\mathrm{t}$ and arbitrary $\left\{\overline{\mathrm{q}}_{\mathrm{i}}^{\mathrm{s}}, \overline{\mathrm{p}}_{\mathrm{i}}^{\mathrm{s}}\right\} \epsilon \mathrm{E}^{6 \mathrm{n}}$ there exists a state function $\mathrm{P}$ such that $\mathrm{P}(\mathrm{t})=\left\{\mathrm{q}_{\mathrm{i}}^{\mathrm{s}}(\mathrm{t}), \mathrm{p}_{\mathrm{i}}^{\mathrm{s}}(\mathrm{t})\right\}=\left\{\overline{\mathrm{q}}_{\mathrm{i}}^{\mathrm{s}}, \overline{\mathrm{p}}_{\mathrm{i}}^{\mathrm{s}}\right\}$.

Assumption 3: The dynamics of the System $\mathrm{S}$ are given by a Hamiltonian function $\mathrm{H}_{\mathrm{n}}=\mathrm{H}_{\mathrm{n}}\left(\left\{\mathrm{q}_{\mathrm{i}}^{\mathrm{s}}, \mathrm{p}_{\mathrm{i}}^{\mathrm{s}}\right\}\right)$ and by a one-parameter group $\left\{\mathrm{U}_{\mathrm{t}}\right\}, \mathrm{t} \in \mathrm{R}^{\#}$, of contact transformations, differentiable with respect to $\mathrm{t}$, so that if $\mathrm{P}$ is an arbitrary given state function then for $\mathrm{t} \in \mathrm{R}^{\#}, \tau \in \mathrm{R}^{\#}$

$$
\mathrm{U}_{\tau} \mathrm{P}(\mathrm{t})=\mathrm{U}_{\tau}\left\{\mathrm{q}_{\mathrm{i}}^{\mathrm{s}}(\mathrm{t}), \mathrm{p}_{\mathrm{i}}^{\mathrm{s}}(\mathrm{t})\right\}=\left\{\mathrm{q}_{\mathrm{i}}^{\mathrm{s}}(\mathrm{t}+\tau), \mathrm{p}_{\mathrm{i}}^{\mathrm{s}}(\mathrm{t}+\tau)\right\}=\mathrm{P}(\mathrm{t}+\tau)
$$

The statistics of the dynamical System are now given, for each value of the parameter $t \epsilon R^{\# \text {, }}$ by a probability distribution $F_{t}$ which for each $t \in R^{*}$ satisfies assumption 1 above so that we may write: $F_{t}(B)=\int_{B} \rho_{t}\left(q_{i}^{s}, p_{i}^{s}\right)(d q d p)_{n}$.

Definition: The System $\mathrm{S}$ in the state $\mathrm{P}(\mathrm{t})$ will be said to be in the Borel set $\mathrm{B}$ at $\mathrm{t}$ if $\mathrm{P}(\mathrm{t})=$ $\left\{\mathrm{q}_{\mathrm{i}}^{\mathrm{s}}(\mathrm{t}), \mathrm{p}_{\mathrm{i}}^{\mathrm{s}}(\mathrm{t})\right\} \epsilon \mathrm{B}$. In these terms $\mathrm{F}_{\mathrm{t}}(\mathrm{B})$ may be referred to as the probability that at $\mathrm{t} \epsilon \mathrm{R} \#$ the System will be in a state in $\mathrm{B}$.

Since the variation of the states $P(t)$, or of the state function $P$, with $t$ is given by assumption 3 , it is possible to describe the related variation of the $F_{t}(B)$ with $t$ as follows. By assumption 3 : $U_{\tau} P(t)=P(t+\tau)$, the states $P(t)$ which are in a Borel set $B$ at $t$ will become states $P(t+\tau)$ in a Borel set $B^{\tau}$ at $t+\tau$. The set $B^{\tau}$ is the set of all points $\left\{\bar{q}_{i}^{s}, \bar{p}_{i}^{s}\right\}$ of $E^{6 n}$ which satisfy $\left\{\bar{q}_{i}^{s}, \bar{p}_{i}^{s}\right\}=\left\{q_{i}^{s}(t+\tau)\right.$, $\left.p_{i}^{s}(t+\tau)\right\}=U_{\tau}\left\{q_{i}^{s}(t), p_{i}^{s}(t)\right\}$ for $\left\{q_{i}^{s}(t), p_{i}^{s}(t)\right\}=\left\{q_{i}^{s}, p_{i}^{s}\right\} \epsilon B$. Since the $U_{t}$ are contact transformations and since the Poincare Invariants under contact transformations include the Lebesgue measure $\mu(B)$ of the Borel sets $B \subset E^{6 n}$, we have $\mu\left(B^{\tau}\right)=\mu(B)$.

For given $t$ and Borel set $B$ with state $\left\{q_{i}^{s}(t), p_{i}^{s}(t)\right\}=\left\{q_{i}^{s}, p_{i}^{s}\right\} \epsilon B$ consider $\rho_{t}\left(q_{i}^{s}, p_{i}^{s}\right) \mu(B)=$ $\rho\left(q_{i}^{s}(t), p_{i}^{s}(t), t\right) \mu(B)$ and similarly for $\tau$ and $B^{\tau}$ with $\left\{q_{i}^{s}(t+\tau), p_{l}^{s}(t+\tau)\right\}=\left\{\bar{q}_{i}^{s}, \bar{p}_{i}^{s}\right\} \epsilon B^{\tau}$ consider $\rho_{t+\tau}\left(\bar{q}_{i}^{s}, \bar{p}_{i}^{s}\right) \mu\left(B^{\tau}\right)=\rho\left(q_{i}^{s}(t+\tau), p_{i}^{s}(t+\tau)\right) \mu\left(B^{\tau}\right)$.

Assumption 4: The functions $\rho_{\mathrm{t}}\left(\mathrm{q}_{\mathrm{i}}^{\mathrm{s}}, \mathrm{p}_{\mathrm{i}}^{\mathrm{s}}\right)=\rho\left(\mathrm{q}_{\mathrm{i}}^{\mathrm{s}}(\mathrm{t}), \mathrm{p}_{\mathrm{i}}^{\mathrm{s}}(\mathrm{t}), \mathrm{t}\right)$ are differentiable with respect to $\mathrm{q}_{\mathrm{i}}^{\mathrm{s}}$, $\mathrm{p}_{\mathrm{i}}^{\mathrm{s}}$ and $\mathrm{t}$ and $\frac{\mathrm{d} \rho}{\mathrm{dt}}$ (which exists by assumed differentiability of the contact transformations $\mathrm{U}_{\mathrm{t}}$ ) is uniformly continuous over each Borel set B.

Then for $\tau=\Delta t$, sufficiently small, and arbitrary closed bounded Borel set $\beta \subset B$ one can write by the law of the Mean:

$$
\begin{aligned}
\rho\left(\mathrm{q}_{\mathrm{i}}^{\mathrm{s}}(\mathrm{t}+\Delta \mathrm{t}), \mathrm{p}_{\mathrm{i}}^{\mathrm{s}}(\mathrm{t}+\Delta \mathrm{t}), \mathrm{t}+\Delta \mathrm{t}\right) \mu\left(\beta^{\Delta \mathrm{t}}\right)=\rho\left(\mathrm{q}_{\mathrm{i}}^{\mathrm{s}}(\mathrm{t}+\Delta \mathrm{t}), \mathrm{p}_{\mathrm{i}}^{\mathrm{s}}(\mathrm{t}+\Delta \mathrm{t}), \mathrm{t}+\Delta \mathrm{t}\right) \mu(\beta) \\
=\rho\left(\mathrm{q}_{\mathrm{i}}^{\mathrm{s}}(\mathrm{t}), \mathrm{p}_{\mathrm{i}}^{\mathrm{s}}(\mathrm{t}), \mathrm{t}\right) \mu(\beta)+\Delta \mathrm{t} \frac{\mathrm{d} \rho}{\mathrm{dt}}\left(\mathrm{q}_{\mathrm{i}}^{\mathrm{s}}\left(\mathrm{t}+\theta_{1} \Delta \mathrm{t}\right), \mathrm{p}_{\mathrm{i}}^{\mathrm{s}}\left(\mathrm{t}+\theta_{2} \Delta \mathrm{t}\right), \mathrm{t}+\theta_{3} \Delta \mathrm{t}\right) \mu(\beta)
\end{aligned}
$$

and using uniform continuity of $\frac{d \rho}{d t}$ one can write

$$
\lim _{\Delta t \rightarrow 0} \frac{F_{t+\Delta t}\left(B^{\Delta t}\right)-F_{t}(B)}{\Delta t}=\int_{B} \frac{d \rho}{d t}\left(q_{i}^{s}, p_{i}^{s}, t\right)(d q d p)_{n} .
$$

Letting $\frac{d F_{t}(B)}{d t}$ represent the left hand expression we make 
Assumption 5: $\frac{\mathrm{dF}_{\mathrm{t}}(\mathrm{B})}{\mathrm{dt}}=0$ for all Borel sets $\mathrm{B}$. From this assumption and continuity of $\frac{\mathrm{d} \rho}{\mathrm{dt}}$ we have

$$
\begin{aligned}
0 & =\frac{\partial \rho}{\partial \mathrm{t}}+\sum_{\mathrm{i}=1}^{3} \sum_{\mathrm{s}=1}^{\mathrm{n}}\left(\frac{\partial \rho}{\partial \mathrm{q}_{\mathrm{i}}^{\mathrm{s}}}\left(\frac{\mathrm{d} \mathrm{q}_{\mathrm{i}}^{\mathrm{s}}}{\mathrm{dt}}\right)+\frac{\partial \rho}{\partial \mathrm{p}_{\mathrm{i}}^{\mathrm{s}}}\left(\frac{\mathrm{d} \mathrm{p}_{\mathrm{i}}^{\mathrm{s}}}{\mathrm{dt}}\right)\right) \\
& =\frac{\partial \rho}{\partial \mathrm{t}}+\sum_{\mathrm{i}, \mathrm{s}}\left(\frac{\partial \rho}{\partial \mathrm{q}_{\mathrm{i}}^{\mathrm{s}}}\left(\frac{\partial \mathrm{H}_{\mathrm{n}}}{\partial \mathrm{p}_{\mathrm{i}}^{\mathrm{s}}}\right)-\frac{\partial \rho}{\partial \mathrm{p}_{\mathrm{i}}^{\mathrm{s}}}\left(\frac{\partial \mathrm{H}_{\mathrm{n}}}{\partial \mathrm{q}_{\mathrm{i}}^{\mathrm{s}}}\right)\right) \\
& =\frac{\partial \rho}{\partial \mathrm{t}}+\left[\rho, \mathrm{H}_{\mathrm{n}}\right] . \quad \text { [Liouville's Theorem] }
\end{aligned}
$$

\section{Derivation of BBGKY Equations}

Consider a subspace $E^{\sigma} \subset E^{6 n}$ where $E^{\sigma}$ is the subspace of $6 \sigma$-tuples $\left\{q_{i}^{\omega}, p_{i}^{\omega}\right\}, \omega=j_{1}, j_{2} \ldots j_{\sigma}$, the $j_{k}$ being $\sigma$ distinct integers from 1 to $n$; for purposes of simplicity in notation take $\omega=1,2, \ldots \sigma$.

For $B_{\sigma}$ a Borel subset of $E^{\sigma}$ let $C_{\sigma}$ be the $6 n$ dimensional "cylinder" on $B_{\sigma}$ i.e., $C_{\sigma}=\left\{\left\{q_{i}^{s}, p_{i}^{s}\right\}\right.$, $i=1,2,3, s=1 \ldots n$; such that for $\left.s=1 \ldots \sigma\left\{q_{i}^{s}, p_{i}^{s}\right\} \subset B_{\sigma}\right\}$. We shall write $C_{\sigma}=B_{\sigma} \times E_{\sigma}^{\prime}$ where $E_{\sigma}^{\prime}=\left\{\left\{q_{i}^{s}, p_{i}^{s}\right\}, s=\sigma+1, \ldots n\right\}$.

Since $C_{\sigma}$ is a Borel subset of $E^{6 n}$ we have as above

$$
\begin{aligned}
F_{t}\left(C_{\sigma}\right) & =\int_{C_{\sigma}} \rho_{t}\left(q_{i}^{s}, p_{i}^{s}\right)(d q d p)_{n} \\
& =\int_{B_{\sigma}}\left[\int_{E_{\sigma}^{\prime}} \rho_{t}\left(q_{i}^{s}, p_{i}^{s}\right)\left(d q_{i}^{\sigma+1} \ldots d p_{i}^{n}\right)\right]\left(d q_{i}^{1} \ldots d p_{i}^{\sigma}\right) .
\end{aligned}
$$

Writing

$$
\rho_{\sigma}\left(q_{i}^{\S}, p_{i}^{s}, t\right)=\int_{E_{\sigma}^{\prime}} \rho_{t}\left(q_{i}^{s}, p_{i}^{s}\right)\left(d q_{i}^{\sigma+1} \ldots d p_{i}^{n}\right)
$$

then

$$
F_{t}\left(C_{\sigma}\right)=\int_{B_{\sigma}} \rho_{\sigma}\left(q_{i}^{s}, p_{i}^{s}, t\right)\left(d q_{i}^{1} \ldots d p_{i}^{\sigma}\right)
$$

The Hamiltonian $H_{n}=H_{n}\left(\left\{q_{i}^{s}, p_{l}^{s}\right\}\right)$ will be of the form in the following assumption:

Assumption 6:-

$$
\mathrm{H}_{\mathrm{n}}=\left(\sum_{\mathrm{k}=1}^{\mathrm{n}} \sum_{\mathrm{i}=1}^{3} \frac{\left(\mathrm{p}_{\mathrm{i}}^{\mathrm{k}}\right)^{2}}{2 \mathrm{~m}}\right)+\mathrm{V}\left(\left\{\mathrm{q}_{\mathrm{i}}^{\mathrm{s}}\right\}\right)
$$

where

$$
\mathrm{V}\left(\left\{\mathrm{q}_{\mathrm{i}}^{\mathrm{s}}\right\}\right)=\sum_{\mathrm{k}=1}^{\mathrm{n}} \sum_{\mathrm{l}>\mathrm{k}}^{\mathrm{n}} \theta_{\mathrm{k}, 1}+\sum_{\mathrm{k}=1}^{\mathrm{n}}\left[\Phi_{\mathrm{w}}^{\mathrm{k}}\left(\left\{\mathbf{q}_{\mathrm{i}}^{\mathrm{s}}\right\}\right)+\Psi_{+}^{\mathrm{k}}\left(\left\{\mathbf{q}_{\mathrm{i}}^{\mathrm{s}}\right\}\right)\right]
$$

with $\theta_{\mathrm{kl}}=\theta_{\mathrm{lk}}=\theta\left(\mathrm{q}_{\mathrm{i}}^{\mathrm{k}}, \mathrm{q}_{\mathrm{i}}^{\mathrm{l}}\right)=$ a symmetric function of $\mathrm{q}_{\mathrm{i}}^{\mathrm{k}}, \mathrm{q}_{\mathrm{i}}^{\mathrm{p}}$; for example: $\theta_{\mathrm{kl}}=\mathrm{e}^{2}\left[\sum_{\mathrm{i}=1}^{3}\left(\mathrm{q}_{\mathrm{i}}^{\mathrm{k}}-\mathrm{q}_{i}^{\mathrm{l}}\right)^{2}\right]^{-1 / 2}$ and $\Phi_{\mathrm{w}}^{\mathrm{k}}\left(\left\{\mathrm{q}_{\mathrm{i}}^{\mathrm{s}}\right\}\right)=$ potential at $\mathrm{k}$ th point (or particle) due to the boundaries (in $\mathrm{E}^{6}$ ) which are assumed to contain the $\mathrm{n}$ points (or particles) and $\Psi_{+}^{\mathrm{k}}\left(\left\{\mathrm{q}_{\mathrm{i}}^{\mathrm{s}}\right\}\right)=$ potential at the $\mathrm{k}$ th point (or particle, or electron) due to a fixed background electric field (produced by + charges).

One can then write

$$
\frac{d F_{t}\left(C_{\sigma}\right)}{d t}=\int_{B_{\sigma}} \int_{E_{\sigma}^{\prime}} \frac{d \rho}{d t}\left(q_{i}^{s}(t), p_{i}^{s}(t), t\right)(d q d p)_{n}
$$




$$
\begin{aligned}
= & \int_{B_{\sigma}} \int_{E_{\sigma}^{\prime}}\left[\frac{\partial \rho}{\partial t}+\sum_{k=1}^{n}\left(\frac{\partial \rho}{\partial q_{i}^{k}}\left(\frac{p_{i}^{k}}{m}\right)-\frac{\partial \rho}{\partial p_{i}^{k}}\left(\frac{\partial V}{\partial q_{i}^{k}}\right)\right)\right](d q d p)_{n} \\
= & \int_{B_{\sigma}} \frac{\partial}{\partial t} \int_{E_{\sigma}^{\prime}} \rho+\int_{B_{\sigma_{k}}} \sum_{k=1}^{\sigma}\left(\frac{p_{i}^{k}}{m}\right) \frac{\partial}{\partial q_{i}^{k}} \int_{E_{\sigma}^{\prime}} \rho+\int_{B_{\sigma}} \int_{E_{\sigma_{\cdot}}^{\prime}} \sum_{k=\sigma+1}^{n} \frac{\partial \rho}{\partial q_{i}^{k}} \frac{p_{i}^{k}}{m} \\
& -\int_{B_{\sigma}} \sum_{k=1}^{\sigma}\left(\sum_{l>k}^{\sigma} \frac{\partial \theta_{k l}}{\partial q_{i}^{k}}+\sum_{l<k} \frac{\partial \theta_{k l}}{\partial q_{i}^{k}}+\frac{\partial \Phi^{k}{ }_{w}}{\partial q_{i}^{k}}+\frac{\partial \Psi_{+}^{k}}{\partial q_{i}^{k}}\right) \frac{\partial}{\partial p_{i}^{k}} \int_{E_{\sigma}^{\prime}} \rho \\
& -\int_{B_{\sigma}} \int_{E_{\sigma}^{\prime}} \sum_{k=1}^{\sigma}\left(\frac{\partial \rho}{\partial p_{i}^{k}}\right) \sum_{l>\sigma}^{n}\left(\frac{\partial \theta_{k l}}{\partial q_{i}^{k}}\right) \\
& -\int_{B_{\sigma}} \int_{E_{\sigma}^{\prime}} \sum_{k=\sigma+1}^{n}\left(\frac{\partial \rho}{\partial p_{i}^{k}}\right)\left(\sum_{l>k}^{n} \frac{\partial \theta_{k l}}{\partial q_{i}^{k}}+\sum_{m<k} \frac{\partial \theta_{m k}}{\partial q_{i}^{k}}+\frac{\partial \Phi_{w}^{k}}{\partial q_{i}^{k}}+\frac{\partial \Psi_{+}^{k}}{\partial q_{i}^{k}}\right) .
\end{aligned}
$$

In the above and following we omit the $(d q, d p)$ notations whenever they are obvious.

Since

$$
\theta_{k l}=\theta_{l k} \text { and } \sum_{l>k}^{\sigma} \frac{\partial \theta_{k l}}{\partial q_{i}^{k}}+\sum_{m<k} \frac{\partial \theta_{m k}}{\partial q_{i}^{k}}=\sum_{\substack{l=1 \\ l \neq k}}^{\sigma} \frac{\partial \theta_{k l}}{\partial q_{i}^{k}}
$$

the above may be written, using the definition of $\rho_{\sigma}$ :

$$
\begin{aligned}
& =\int_{B_{\sigma}}\left[\frac{\partial \rho_{\sigma}}{\partial t}+\sum_{k=1}^{\sigma}\left(\frac{\partial \rho_{\sigma}}{\partial q_{i}^{k}}\right)\left(\frac{p_{i}^{k}}{m}\right)-\sum_{k=1}^{\sigma}\left(\frac{\partial \rho_{\sigma}}{\partial p_{i}^{k}}\right)\left(\sum_{\substack{l=1 \\
l \neq k}}^{\sigma} \frac{\partial \theta_{k l}}{\partial q_{i}^{k}}\right)\right] \\
& -\int_{B_{\sigma}} \sum_{k=1}^{\sigma} \frac{\partial}{\partial p_{i}^{k}} \cdot \sum_{l>k}^{n} \int_{E_{\sigma}^{\prime}}\left(\frac{\partial \theta_{k l}}{\partial q_{i}^{k}}\right) \rho-\int_{B_{\sigma}} \sum_{k=1}^{\sigma} \frac{\partial \rho_{\sigma}}{\partial p_{i}^{k}}\left(\frac{\partial \Phi_{w}^{k}}{\partial q_{i}^{k}}+\frac{\partial \Psi_{+}^{k}}{\partial q_{i}^{k}}\right) \\
& +\int_{B_{\sigma}} \int_{E_{\sigma}^{\prime}} \sum_{k=\sigma+1}^{n}\left(\frac{\partial \rho}{\partial q_{i}^{k}}\right)\left(\frac{p_{i}^{k}}{m}\right) \\
& -\int_{B_{\sigma}} \int_{E_{\sigma}^{\prime}} \sum_{k=\sigma+1}^{n}\left(\frac{\partial \rho}{\partial p_{i}^{k}}\right)\left(\sum_{\substack{l=1 \\
l \neq k}}^{n} \frac{\partial \theta_{k l}}{\partial q_{i}^{k}}+\frac{\partial \Phi_{w}^{k}}{\partial q_{i}^{k}}+\frac{\partial \Psi_{+}^{k}}{\partial q_{i}^{k}}\right)
\end{aligned}
$$

and one can abbreviate the notation for these five integrals as:

$$
=I(1)-I(2)-I(3)+I(4)-I(5) \text {. }
$$

Now in the above notation for $E^{\sigma}=\left\{\left\{q_{i}^{k}, p_{i}^{k}\right\} ; k=1 \ldots \sigma\right\}$ letting $E^{(l)}=\left\{\left\{q_{i}^{k}, p_{i}^{k}\right\} ; k=l\right\}$ we set $E^{\sigma, l}=E^{\sigma} \dot{+} E^{(l)}$, that is, the minimum subspace containing the subspaces $E^{\sigma}, E^{(l)}$. Also, as above, let $E_{\sigma}^{\prime}=$ the complementary subspace of $E^{\sigma}$ in $E^{6 n}$ consisting of all $6(n-\sigma)$-tuples $\left\{q_{i}^{k}, p_{i}^{k}\right\}$ where $k=\sigma+1, \sigma+2, \ldots n$ and $i=1,2,3$ and let $E_{\sigma, l}^{\prime}=$ the complementary subspace of $E^{\sigma, l}$ in $E^{6 n}$ consisting of all $6(n-\sigma-1)$-tuples $\left\{q_{i}^{k}, p_{i}^{k}\right\}$ where $k \neq l, \sigma+1 \leqslant k \leqslant n$ and $i=1,2,3$. Also similar to the definition of $\rho_{\sigma}$ above we shall let:

$$
\rho_{\sigma, l}\left(q_{i}^{s}, p_{i}^{s}, t\right)=\int_{E_{\sigma, l}^{\prime}} \rho\left(q_{i}^{s}, p_{i}^{s}, t\right)(d q d p)_{\sigma, l}^{\prime}
$$

where $(d q d p)_{\sigma, l}^{\prime}$ represents the usual "volume" element in $E_{\sigma, l}^{\prime}$. 
Furthermore we now make the following:

Assumption 7: $\rho\left(\mathrm{q}_{\mathrm{i}}^{\mathrm{s}}, \mathrm{p}_{\mathrm{i}}^{\mathrm{s}}, \mathrm{t}\right)$ is a function symmetric with respect to the indices $\mathrm{s}$.

It is clear then that the functions $\rho_{\sigma+1}\left(q_{i}^{s}, p_{i}^{s}, t\right)=\rho_{\sigma, \sigma+1}\left(q_{i}^{s}, p_{i}^{s}, t\right)$ and $\rho_{\sigma, 1}\left(q_{i}^{s}, p_{i}^{s}, t\right)$ differ only in that the coordinates $q_{i}^{\sigma+1}, p_{i}^{\sigma+1}$ occurring in $\rho_{\sigma+1}$ are replaced by $q_{i}^{l}, p_{i}^{l}$ in $\rho_{\sigma, l}$. Thus

$$
\begin{aligned}
\mathrm{I}(2) & =\int_{\mathrm{B}_{\sigma}} \sum_{\mathrm{k}=1}^{\sigma} \frac{\partial}{\partial \mathrm{p}_{\mathrm{i}}^{\mathrm{k}}} \sum_{\mathrm{l}>\mathrm{k}}^{\mathrm{n}} \int_{\mathrm{E}_{\sigma}^{\prime}} \frac{\partial \theta_{\mathrm{kl}}}{\partial \mathrm{q}_{\mathrm{i}}^{\mathrm{k}}} \rho \\
& =\int_{\mathrm{B}_{\sigma}} \sum_{\mathrm{k}=1}^{\sigma} \frac{\partial}{\partial \mathrm{p}_{\mathrm{i}}^{\mathrm{k}}} \sum_{\mathrm{l}>\mathrm{k}}^{\mathrm{n}} \int_{\mathrm{E}^{(1)}} \frac{\partial \theta_{\mathrm{kl}}}{\partial \mathrm{q}_{\mathrm{i}}^{\mathrm{k}}} \int_{\mathrm{E}_{\sigma, \sigma}^{\prime} \rho} \rho\left(\mathrm{q}_{\mathrm{i}}^{\mathrm{s}}, \mathrm{p}_{\mathrm{i}}^{\mathrm{s}}, \mathrm{t}\right)(\mathrm{dqdp})_{\sigma, 1}^{\prime}\left(\mathrm{dq}_{\mathrm{i}}^{1}, \mathrm{dp}_{\mathrm{i}}^{1}\right) \\
& =\int_{\mathrm{B}_{\sigma}} \sum_{\mathrm{k}=1}^{\sigma} \sum_{\mathrm{l}>\mathrm{k}}^{\mathrm{n}} \int_{E}^{(1)} \frac{\partial \theta_{\mathrm{kl}}}{\partial \mathrm{q}_{\mathrm{i}}^{\mathrm{k}}} \frac{\partial}{\partial \mathrm{p}_{\mathrm{i}}^{\mathrm{k}}}\left(\rho_{\sigma, 1}\left(\mathrm{q}_{\mathrm{i}}^{\mathrm{s}}, \mathrm{p}_{\mathrm{i}}^{\mathrm{s}}, \mathrm{t}\right)\right) \mathrm{dq}_{\mathrm{i}}^{1} \mathrm{dp}_{\mathrm{i}}^{1} \\
& =\int_{\mathrm{B}_{\sigma}} \sum_{\mathrm{k}=1}^{\sigma}(\mathrm{n}-\sigma) \int_{\mathrm{E}^{(\sigma+1)}} \frac{\partial \theta_{\mathrm{k}, \sigma+1}}{\partial \mathrm{q}_{\mathrm{i}}^{\mathrm{k}}} \frac{\partial \rho_{\sigma+1}}{\partial \mathrm{p}_{\mathrm{i}}^{\mathrm{k}}}\left(\mathrm{dq}_{\mathrm{i}}^{\sigma+1} \mathrm{dp}_{\mathrm{i}}^{\sigma+1}\right)
\end{aligned}
$$

since

$$
\int_{\mathbf{E}^{(\sigma+1)}} \frac{\partial \theta_{\mathbf{k}, \sigma+1}}{\partial \mathbf{q}_{\mathrm{i}}^{\mathrm{k}}} \frac{\partial}{\partial \mathbf{p}_{\mathrm{i}}^{\mathrm{k}}}\left(\rho_{\sigma, \sigma+1}\right)=\int_{\mathbf{E}^{(1)}} \frac{\partial \theta_{\mathrm{kl}}}{\partial \mathrm{q}_{\mathrm{i}}^{\mathrm{k}}} \frac{\partial}{\partial \mathbf{p}_{\mathrm{i}}^{\mathrm{k}}}\left(\rho_{\sigma, 1}\right) .
$$

Suppose now that the states of the System are restricted by:

Assumption 8: There exists a closed bounded subset $\Sigma^{3 \mathrm{n}}$ of $\mathrm{E}^{3 \mathrm{n}}$ such that the probability density function $\rho\left(\mathrm{q}_{\mathrm{i}}^{\mathrm{s}}, \mathrm{p}_{\mathrm{i}}^{\mathrm{s}}, \mathrm{t}\right)$ is identically 0 for all $\left(\mathrm{q}_{\mathrm{i}}^{\mathrm{s}}, \mathrm{p}_{\mathrm{i}}^{\mathrm{s}}, \mathrm{t}\right)$ such that $\left\{\mathrm{q}_{\mathrm{i}}^{\mathrm{s}}\right\} \mathrm{s}=1 \ldots \mathrm{n}, \mathrm{i}=1,2,3$ is a point on the boundary of $\Sigma^{3 \mathrm{n}}$ or outside $\Sigma^{3 \mathrm{n}}$. This is equivalent to saying that for any $\mathrm{t}$ and any Borel set $\mathrm{B}$ with $\mathrm{B} \cap \mathrm{E}^{3 \mathrm{n}}$ in the closure of the complement of $\Sigma^{3 \mathrm{n}}$, the probability, $\left.\mathrm{F}_{\mathrm{t}} \mathrm{B}\right)$, that the System is in a state in $\mathrm{B}$ is 0 .

We shall also take the boundary of $\Sigma^{3 n}$ to be the boundary pertaining to the potential functions $\Phi_{w}^{k}$ of assumption 6 .

One can then write:

$$
I(4)=\int_{B_{\sigma}} \int_{E_{\sigma}^{\prime}} \sum_{k=\sigma+1}^{n}\left(\frac{\partial \rho}{\partial q_{i}^{k}}\right)\left(\frac{p_{i}^{k}}{m}\right)=\int_{B_{\sigma}} \sum_{k=\sigma+1}^{n} \int_{E_{\sigma, k}^{\prime}}\left[\int_{-\infty}^{\infty}\left(\frac{p_{i}^{k}}{m}\right)\left[\int_{-\infty}^{\infty} \frac{\partial \rho}{\partial q_{i}^{k}} d q_{i}^{k}\right] d p_{i}^{k}\right](d q d p)_{\sigma, k}^{\prime}
$$

and

$$
\int_{-\infty}^{\infty} \frac{\partial \rho}{\partial q_{i}^{k}} d q_{i}^{k}=0 \text { by virtue of assumption } 8 \text { hence } I(4)=0
$$

If also one makes:

Assumption 9: For any given $\mathrm{k}=1 \ldots \mathrm{n} \quad \rho\left(\mathrm{q}_{\mathrm{i}}^{\mathrm{s}}, \mathrm{p}_{\mathrm{i}}^{\mathrm{s}}, \mathrm{t}\right) \rightarrow 0$ as $\mathrm{p}_{\mathrm{i}}^{\mathrm{k}} \rightarrow \pm \infty$ then $I(5)=0$ by the same type of argument.

Finally from assumption $5 \frac{d F_{+}\left(C_{\sigma}\right)}{d t}=0$ for any $C_{\sigma}$ hence for any $B_{\sigma} 0=I(1)-I(2)-I(3)$ and since the integrands are assumed continuous we get the BBGKY hierarchy equations:

$$
\begin{aligned}
\frac{\partial \rho_{\sigma}}{\partial t}+\sum_{k=1}^{\sigma}\left(\frac{\partial \rho_{\sigma}}{\partial q_{i}^{k}}\right)\left(\frac{p_{i}^{k}}{m}\right)-\sum_{k=1}^{\sigma}\left(\frac{\partial \rho_{\sigma}}{\partial p_{i}^{k}}\right)\left(\sum_{\substack{l=1 \\
l \neq k}}^{\sigma} \frac{\partial \theta_{k l}}{\partial q_{i}^{k}}+\frac{\partial \Phi_{w}^{k}}{\partial q_{i}^{k}}\right. & \left.+\frac{\partial \Psi_{+}^{k}}{\partial q_{i}^{k}}\right) \\
& =\sum_{k=1}^{\sigma}(n-\sigma) \int_{E^{(\sigma+1)}} \frac{\partial \theta_{k, \sigma+1}}{\partial q_{i}^{k}} \frac{\partial \rho_{\sigma+1}}{\partial p_{i}^{k}}\left(d q_{i}^{\sigma+1} d p_{i}^{\sigma+1}\right) .
\end{aligned}
$$


If one takes the Hamiltonian $H_{\sigma}=H_{\sigma}\left(\left\{q_{i}^{s}, p_{i}^{s}\right\}\right) s=1 \ldots \sigma$ to be

$$
H_{\sigma}=\sum_{k=1}^{\sigma}\left(\sum_{i=1}^{3} \frac{\left(p_{l}^{k}\right)^{2}}{2 m}+\sum_{l>k}^{\sigma} \theta_{k l}+\Phi_{w}^{k}\left(q_{i}^{k}\right)+\Psi_{+}^{k}\left(q_{i}^{k}\right)\right)
$$

then these equations become

$$
\frac{\partial \rho_{\sigma}}{\partial t}+\left[\rho_{\sigma} H_{\sigma}\right]=\sum_{k=1}^{\sigma}(n-\sigma) \int_{E^{(\sigma+1)}} \frac{\partial \theta_{k, \sigma+1}}{\partial q_{i}^{k}} \frac{\partial \rho_{\sigma+1}}{\partial p_{i}^{k}}\left(d q_{i}^{\sigma+1} d p_{i}^{\sigma+1}\right)
$$

The conditions under which these BBGKY equations are valid are thus explicitly given by the assumptions 1 through 9.

\section{More General Case}

The preceding discussion is conditioned by its restriction to Systems of $6 n$ observables $Q_{i}, P_{i} \quad i=1 \ldots 3 n$, that is, to exactly $n$ points (particles) in $E^{6}$. Therefore the functions $F_{T}(B)=F(B, t)$ are, in this sense, conditional probability distributions. A somewhat more general situation may be treated as outlined briefly in the following.

Let $E^{\omega}$ be the linear space of all infinite double sequences:

$$
\left\{q_{1}^{1}, q_{2}^{1}, q_{3}^{1}, q_{1}^{2}, q_{2}^{2}, \ldots p_{1}^{1}, p_{2}^{1}, p_{3}^{1}, p_{1}^{2}, p_{2}^{2}, \ldots\right\}=\left\{q_{i}^{s}, p_{i}^{s}\right\} i=1,2,3 s=1,2, \ldots . .
$$

If $E^{6}=\left\{q_{i}, p_{i}\right\} i=1,2,3$ then $E^{\omega}$ may also be regarded as the infinite direct product:

$$
E^{\omega}=E^{6} \times E^{6} \times E^{6} \times \ldots
$$

Similarly writing as above $E^{\sigma}=\left\{\left\{q_{i}^{\varsigma}, p_{1}^{s}\right\} ; i=1,2,3 ; s=1,2 \ldots \sigma\right\}$ and $B^{\sigma}$ for a Borel subset of $E^{\sigma}$ then for $m \geqq \sigma$ we denote the cylinders $C_{\sigma}^{m}$ in $E^{m}$ and $C_{\sigma}^{\omega}$ in $E^{\omega}$ by: $C_{\sigma}^{m}=B_{\sigma} \times E_{\sigma}^{m^{\prime}}$ where

$E_{\sigma}^{m^{\prime}}=\left\{\left\{q_{i}^{s}, p_{i}^{s}\right\} ; s=\sigma+1, \ldots . m\right\}$ and $C_{\sigma}^{\omega}=B_{\sigma} \times E_{\sigma}^{\omega^{\prime}}$ where $E_{\sigma}^{\omega^{\prime}}=\left\{\left\{q_{i}^{s}, p_{i}^{s}\right\} ; s=\sigma+1, \ldots\right\}$

If the System may vary stochastically with respect to $t$ we may let $P_{m}(t)$ be the probability that, for $t \epsilon R$, there are $m$ points (particles) in $E^{6}$ or equivalently that the System consists of $6 m$ observables $Q_{i}, P_{i}$. Also let $F_{\sigma}^{m}\left(C_{\sigma}^{m}, t\right)$ be the conditional probability that, if there are $6 m$ observables in the System, then this System will be in a state $P=\left\{q_{i}^{s}, p_{i}^{s}\right\}$ such that the $6 \sigma$-tuple $\left\{q_{i}^{s}, p_{i}^{s}\right\} s=1 \ldots \sigma$ will be in $B_{\sigma}$ at $t$. Then the probability that the System will be in a state $P=\left\{q_{i}^{s}, p_{i}^{s}\right\}$ which is in $B_{\sigma}$ at $t$ is given by: $F_{\sigma}^{\omega}\left(C_{\sigma}^{\omega}, t\right)=\sum_{m \geqslant \sigma} P_{m}(t) F_{\sigma}^{m}\left(C_{\sigma}^{m}, t\right)$.

If for each $m$ the distribution $F_{\sigma}^{m}\left(C_{\sigma}^{m}, t\right)$ is absolutely continuous with respect to Lebesgue measure in $E^{m}$ then there exists as above a measurable function $\rho_{\sigma}^{m}$ such that

$$
F_{\sigma}^{m}\left(C_{\sigma}^{m}, t\right)=\int_{C_{\sigma}} \rho_{\sigma}^{m}\left(q_{i}^{s}, p_{i}^{s}, t\right)(d q d p)_{m}
$$

Furthermore $F_{\sigma}^{\omega}\left(C_{\sigma}^{\omega}, t\right)$ will also be absolutely continuous with respect to the product measure $E^{\omega}$ and $F_{\sigma}^{\omega}\left(C_{\sigma}, t\right)=\int_{C_{\sigma}} \rho_{\sigma}^{\omega}\left(q_{i}^{\S}, p_{i}^{s}, t\right)(d q d p)_{\omega}$ where $\rho_{\sigma}^{\omega}$ is a function measurable with respect to the product measure in $E^{\omega}$.

Since $F_{\sigma}^{\omega}\left(C_{\sigma}^{\omega}, t\right)=\sum_{m \geqslant \sigma} P_{m}(t) F_{\sigma}^{m}\left(C_{\sigma}^{m}, t\right)$ the sequence $\sum_{m \geqslant \sigma}^{n} P_{m}(t) \rho_{\sigma}^{m}\left(q_{i}^{s}, p_{i}^{s}, t\right)$, as $n \rightarrow \infty$, converges in measure to $\rho_{\sigma}^{\omega}\left(q_{i}^{s}, p_{i}^{s}, t\right)$ and under proper assumptions on the uniform continuity of the 
derivatives of the terms of the sequence one may relate the hierarchy equations for the $\rho_{\sigma}^{\omega}$ to the above derived corresponding relations for the individual $\rho_{\sigma}^{m}$ occurring in the terms of the sequence.

\section{Summary}

The preceding derivation of the BBGKY hierarchy equations and the specific assumptions on which it is based are not restricted to plasma kinetics. As indicated in the introduction the treatment is essentially probabilistic and nearly all the assumptions are probabilistic in nature. The excepted nonprobabilistic assumptions refer to the existence of a Hamiltonian and an associated contact transformation group, both of which are more general than the requirements of plasma kinetics.

\section{References}

Halmos, P. R. (1950), Measure Theory (D. Van Nostrand Co., New York, N.Y.).

Kakutani, S. (1943), Notes on infinite product measure spaces I, Proc. Imp. Acad. Tokyo 19.

Montgomery, D. C., and D. A. Tidman (1964), Plasma Kinetic Theory (McGraw-Hill Book Co., Inc., New York, N.Y.).

Zaanen, A. C. (1967), Integration (John Wiley \& Sons, Inc., New York, N.Y.).

Khinchin, A. I. (1949), Mathematical Methods of Statistical Mechanics (Dover Publications, Inc., New York, N.Y.).

Liouville, J. (1838), Journal de Mathematique 3.

Lorentz, J. (1887), Wiener Sitzungsberichte 95 (2).

(Paper 72B2-266) 